Voix et Images

voixetimages

\title{
Ces jeunes contestataires des années 30 : Albert Pelletier-Alfred DesRochers (1929-1936)
}

\section{Richard Giguère}

Volume 16, numéro 1 (46), automne 1990

Les correspondants littéraires d'Alfred DesRochers

URI : https://id.erudit.org/iderudit/200870ar

DOI : https://doi.org/10.7202/200870ar

Aller au sommaire du numéro

Éditeur(s)

Université du Québec à Montréal

ISSN

0318-9201 (imprimé)

1705-933X (numérique)

Découvrir la revue

Citer cet article

Giguère, R. (1990). Ces jeunes contestataires des années 30 : Albert Pelletier-Alfred DesRochers (1929-1936). Voix et Images, 16(1), 8-25.

https://doi.org/10.7202/200870ar d'utilisation que vous pouvez consulter en ligne.

https://apropos.erudit.org/fr/usagers/politique-dutilisation/ 


\title{
Ces jeunes contestataires des années 30: Albert Pelletier- Alfred DesRochers (1929-1936)
}

\section{par Richard Giguère, Université de Sherbrooke}

\author{
À la mémoire d'Albert Pelletier \\ Il fut pour nous le connétable de l'esprit, \\ Le héraut de la phrase intègre et du mot juste, \\ Sans pourtant les réduire au sommier de Procuste \\ En ce sièclé où le fait l'emporte sur l'esprit. \\ Tel un chêne géant où l'aigle trouve abri, \\ De son ombre élaguant la broussaille et l'arbuste, \\ Il fit un sous-bois clair à la pensée augusté \\ Où les nouveaux venus s'avancent aujourd'hui. \\ Mais il était meilleur ami que juge encore \\ Et son cœur tempérait le vocable sonore \\ Que son esprit lucide eût d'abord professé. \\ J'entends déjà sur moi la mort battre des ailes; \\ Je vois l'autre versant du sommet dépassé \\ Où s'uniront enfin nos ombres fraternelles.
}

Alfred DesRochers (novembre 1971)

Alfred DesRochers a raison d'utiliser le titre de connétable de l'esprit pour parler de son ami Albert Pelletier, qui fut sans doute le plus puissant et le plus redouté de tous les critiques littéraires des années trente. C'est avec raison aussi que DesRochers évoque d'une part le héraut de la phrase intègre et du mot juste et d'autre part le juge au vocable sonore, car à lire la centaine de lettres que l'auteur de Carquois adressa au poète de l'Orford, on se rend compte que Pelletier aiguisait sa plume comme une lame avant d'écrire le compte rendu d'un livre: Une fois réchauffé, il frappait les mots et les sentences comme un soldat d'élite frappe de l'épée un soldat ennemi. Quant aux vers Tel un chêne géant [...] / Il fit un sous-bois clair à la pensée auguste / Où les nouveaux venus s'avancent aujourd'hui, il nous est difficile, à cinquante ans de distance, d'en saisir exactement le sens. Il importe donc de relire les œuvres critiques de Pelletier, 
puis de lire attentivement sa correspondance avec DesRochers et de replacer tout cela dans le contexte littéraire, culturel et sociopolitique de l'entre-deux-guerres avant de nous prononcer sur ce sonnet écrit par DesRochers, en 1971, à la mémoire d'Albert Pelletier.

Rappelons d'abord qu'au moment où ils commencent à échanger des lettres, en 1929, nos protagonistes sont deux jeunes écrivains actifs, mais encore peu connus des milieux littéraires montréalais et québécois. Albert Pelletier (1896-1971) a trente-trois ans et vit à Montréal, dans un modeste logement de la rue Saint-Hubert, avec son épouse et deux enfants en bas âge (Gilles et Denise, les futurs acteurs de la scène et de la télévision). Fils de cultivateur et notaire de profession, il est registraire adjoint au Bureau d'enregistrement de Montréal depuis 1927, poste qu'il occupe grâce à un cours classique au collège de Sainte-Anne de la Pocatière et à des études de droit à l'université Laval et à l'Université de Montréal. De retour dans la métropole après son mariage et un court séjour à Saint-Jovite, il commence à signer des comptes rendus dans les pages de la Revue moderne à la fin des années vingt. Il écrit au début des années trente pour le Canada d'Olivar Asselin et publie ses deux recueils de critiques, Carquois (1931) et Égrappages (1933, prix David), aux éditions de la Librairie d'action canadienne-française/Albert Lévesque. C'est pendant les années de correspondance avec DesRochers qu'il met sur pied avec Lucien Parizeau les Éditions du Totem (1933) et qu'il crée par la suite une revue de combat, les Idées (1935-1939).

Quant à DesRochers (1901-1978), né à Saint-Élie d'Orford dans une famille de cultivateurs, même s'il est de cinq ans le cadet de Pelletier, il a déjà exercé plusieurs métiers à la fin des années vịngt, dont celui de reporter à la Tribune de Sherbrooke (1925-1927), après trois années d'humanités classiques (1918-1921) au Collège séraphique de Trois-Rivières. Il remet sur pied l'hebdomadaire l'Étoile de l'Est de Coaticook en 1927, puis revient à la Tribune à titre d'agent de publicité en 1928, l'année même où il lance à compte d'auteur son premier recueil, l'Offrande aux vierges folles (tirage limité à 150 exemplaires, novembre 1928).

\section{Les samedis soirs au 3681 de la rue Saint-Hubert}

Les premières lettres ne sont pas tout à fait claires là-dessus, mais il semble bien que ce soit ce recueil qui ait marqué le début de la correspondance, DesRochers ayant en janvier ou février 1929 par l'intermédiaire du poète Robert Choquette, alors directeur des pages littéraires de la Revue moderne - envoyé un exemplaire de l'Offrande à Pelletier. Chose certaine, celui-ci écrivit un compte rendu très positif du recueil pour la livraison de mars 1929 de la revue de Madeleine Huguenin. Or, dès le début de février, le critique montréalais adressait un petit mot au poète de Sherbrooke, l'invitant, 
avec madame Desrochers, à participer à une soirée qui devait se tenir chez lui, au 3681 de la rue Saint-Hubert, le samedi 2 février. Les DesRochers ne pouvant se rendre à Montréal ce soir-là, Pelletier écrivit par retour du courrier pour les inviter à une autre soirée, le jeudi 7 février, au lendemain de l'annonce des résultats du concours des prix d'Action intellectuelle. Il confiait même à DesRochers la mission d'amener avec lui celles qu'il croyait être ses "élèves", en poésie, Jovette Bernier et Éva Senécal.

Cette soirée n'est que la première d'une longue série de réunions littéraires (les samedis soirs) tenues chez les Pelletier: elles eurent lieu une ou deux fois par mois au début des années trente, puis quatre à six fois par année par la suite. Dès l'hiver 1929 et assez souvent en 1930 et en 1931, puis beaucoup moins fréquemment de 1932 à 1935, les DesRochers se joignent à un groupe d'écrivains (Jovette Bernier, Roger Brien, Robert Choquette, Emile Coderre (Jean Narrache), Françoise Gaudet, Claude-Henri Grignon (Valdombre), Alice Lemieux, Clémént Marchand, Éva Senécal, Medjé Vézina), de journalistes (Olivar Asselin à l'occasion, Lucien Parizeau, Edmond Turcotte, Roger Lemelin plus tard) et de critiques (Willie Chevalier, Henri Girard) qui se rencontrent de façon informelle pour des soirées et même des nuits de discussion. Des réunions surtout "antisnobistes", insiste Pelletier: tous les auteurs qui ont signé un témoignage dans l'anthologie d'Albert Pelletier, préparée par Lucien Parizeau pour les Écrits du canada français en $1972^{1}$, ' s'accordent là-dessus. Dans le texte d'introduction, Parizeau affirme que c'est l'éclectisme des goûts du critique montréalais qui fut à l'origine de ces réunions, et que ces soirées

ne furent jamais les messes séculières d'une chapelle, mais plutôt les occasions d'affrontement de quelques esprits, au fond très différents les uns des autres, mais tous animés du besoin de penser à leur aise et de débattre le destin du monde jusqu'au petit matin ${ }^{2}$.

L'expression à retenir est écrite en toutes lettres par Parizeau: penser à leur aise. Le groupe qui se réunit chez Pelletier, et en particulier Valdombre, Alfred DesRochers, Clément Marchand, Jean Narrache, Jovette Bernier et Medjé Vézina, se compose d'hommes et de femmes de lettres et d'action qui veulent penser et s'exprimer

1 Voir "Albert Pelletier (1895-1971)», Écrits du Canada français (désormais ÉCF), $n^{\circ} 34,1972$, p. 9-122. Cette anthologie, préparée par Lucien Parizeau, comprend une introduction, des souvenirs et témoignages de DesRochers, Jovette Bernier, Medjé Vézina, Willie Chevalier, Robert Choquette, Albert Lévesque, Françoise Gaudet-Smet, Clément Marchand et Roger Lemelin, des extraits tirés de Carquois et d'Égrappages, une bonne sélection d'articles/choisis dans les Idées et quelques comptes rendus de livres des années trente.

2 Lucien Parizeau, "Introduction ", ibid., p. 14. 
librement. Ces gens viennent non seulement de Montréal, mais de la région des Laurentides, de Québec, de Sherbrooke, de Trois-Rivières, et acquièrent au cours de ces années, entre autres en fréquentant le salon des Pelletier ${ }^{3}$, une réputation d'esprits indépendants, de polémistes et de francs-tireurs. Il ne s'agit pas de révolutionnaires qui veulent tout détruire pour repartir à zéro: cela est difficilement concevable dans le Québec des années trente, sauf pour quelques rares exceptions. Pelletier, DesRochers et Dantin, par exemple, discutent dans leur correspondance des mérités des différents régimes politiques et systèmes économiques, flirtent avec le socialisme et le communisme (Dantin-DesRochers), mais ils brassent des idées sans fomenter la révolution. Avant tout, les Pelletier, DesRochers, Valdombre et Jean Narrache prônent des réformes dans le domaine de la culture (à leur avis coupée de la réalité), des valeurs matérielles omniprésentes, des valeurs nationales (slogans passéistes et stériles), du système d'enseignement figé et concentré entre les mains du clergé. En somme, ils contestent dans le but de réformer.

L'esprit du groupe qui se réunit chez Pelletier, tel que le définissent Parizeau et les acteurs ou les témoins de l'époque, donne une bonne idée de l'esprit de la correspondance Pelletier-DesRochers. Les lettres sont la plupart du temps amicales, mais Pelletier hausse souvent le ton et alors le côté polémiste et même pamphlétaire du jeune critique prend le pas sur la discussion objective et la réflexion nuancée. DesRochers se laisse rarement emporter par l'argumentation, mais ne cède pas facilement un pouce de terrain et reproche même à Pelletier sa sévérité et ses excès de langage. Les prises de bec sont rares lorsque les deux correspondants demeurent sur le terrain des définitions et des principes de la littérature et de la critique littéraire, mais aussitôt qu'ils abordent des sujets controversés comme l'enseignement dans les collèges classiques, la conception de l'autorité politique, la langue et la culture "canadiennes" par opposition à la langue et la culture françaises, il suffit de quelques étincelles pour que la discussion s'enflamme.

Il faut dire aussi que DesRochers et Pelletier échangent un grand nombre, et souvent de longues lettres, à une époque où les deux hommes sont très sollicités, autant dans leur travail professionnel et leur carrière littéraire que dans leur vie personnelle. Le fonds

3 Précisons qu'Albert Pelletier n'est pas le seul à tenir des réunions littéraires au cours des années trente. Albert Lévesque, qui demeure en face des Pelletier, rue Saint-Hubert, reçoit quelquefois chez lui, de même qu'Émile Coderre. Il y a également les célèbres "leçons» de français écrit qu'Olivar Asselin donne à des écrivains et à des journalistes, à son bureau du journal le Canada, le dimanche matin. Enfin, les soirées et les réunions ne se tiennent pas uniquement à Montréal. DesRochers organise quelques mémorables réunions d'écrivains à Sherbrooke (20, rue Georges) alors que Clément Marchand lance des invitations de Trois-Rivières. 
DesRochers, déposé par le poète et sa famille aux Archives nationales du Québec à Sherbrooke, contient 90 lettres de Pelletier, allant d'une simple carte à des épîtres de quatre pages, datées du 4 février 1929 au 23 août 1936. Les années les plus importantes sont 1930 et 1931 (16 lettres de Pelletier par année), 1933 (21 lettres) et 1934 (13 lettres), la moyenne générale atteignant douze lettres par correspondant par année. Le critique montréalais écrit d'abord des lettres à la main (Cher M. DesRochers), puis tape directement à la machine (Mon cher poète) sur du papier à lettres à en-tête du Bureau d'enregistrement de la division de Montréal. Avec le temps, Pelletier utilise aussi du papier grand format $(21 \times 35 \mathrm{~cm})$ et allonge ses lettres à deux trois pages (simple interligne et recto verso) ${ }^{4}$. La meilleure année (1933) contient des lettres de toutes les longueurs et de tous les formats, alors que 1934 voit apparaître le beau papier à en-tête des Éditions du Totem. Par contre, le fonds ne possède que 9 des 90 lettres de DesRochers, en fait des copies carbone assez espacéès entre elles (de 1930 à 1935). Heureusement pour nous, le poète a choisi de faire des doubles des lettres les plus importantes qu'il a adressées à Pelletier, et qui traduisent bien la teneur et le ton de l'ensemble de leur correspondance ${ }^{5}$.

\section{Théorie et critique littéraires}

Les sujets les plus discutés par les deux jeunes critiques et écrivains, du moins dans la première partie de leur correspondance, sont ceux de la théorie de la littérature - de la poésie en particulier - et de la conception de la critique littéraire et de son rôle. Au plus fort de la Crise, en 1933-1934, Pelletier et DesRochers échangeront des lettres sur les mérites respectifs des régimes politiques et des systèmes monétaires et, de 1934 à 1936, ce sont les problèmes concrets de la fabrication, de la mise en marché et de la diffusion des livres et des revues qui retiennent leur attention. Mais au début des années trente, ils en sont encore à énoncer leur conception respective de la littérature et de la critique, en tâchant de n'être pas trop en désaccord.

4 Il faut imaginer Albert Pelletier - selon le témoignage de Lucien Parizeau tapant ses lettres directement à la machine à écrire, le soir, sur la table de la salle à manger de son modeste logement, et grillant cigarette après cigarette jusque tard dans la nuit.

5 DesRochers tapait aussi ses lettres directement à la machine, selon la bonne vieille méthode "à deux doigts" des journalistes de l'époque, sur du papier à lettres format usuel (21 x $28 \mathrm{~cm}$ ) ou grand format $(21 \times 35 \mathrm{~cm})$. La grande majorité de ces lettres de deux, trois, ou même quatre et cinq pages sont malheureusement perdues à jamais, si l'on se fie au témoignage de Pelletier lui-même, qui écrivait à Lucien Parizeau peu de temps avant sa mort: Je te dis tout de suite [...] que ma correspondance, tant avec Louis Dantin qu'avec tous les autres, a disparu dans notre déménagement de Saint-Marc-sur-Richelieu à Montréal, en 1965 (je pense que notre servante a voulu faire du zèle et qu'elle a fait passer par la flamme du foyer deux caisses de correspondance que javais pris le soin de ranger et de classer par liasses alphabétiques). (Lucien Parizeau, loc cit., p. 16) 
Pourtant, avec le recul, il nous apparaît clairement aujourd'hui qu'ils ne pouvaient pas arriver à s'entendre sur le sujet. Pelletier affirme dès le départ qu'en littérature, avant le style, il y a d'abord le fond: une idée, une impression, un sentiment, une passion et que même en poésie, [...] le fond prime tout (3 décembre 1929). Il revient régulièrement sur cette question de la prépondérance du fond sur la forme et de l'utilité immédiate d'un roman ou d'un recueil de poèmes. La preuve: les sonnets d'Ä l'ombre de l'Orford n'ont-ils pas été écrits pour glorifier le travail manuel (30 mars 1934)? Le fait est que DesRochers pense exactement le contraire et, après un certain temps, il ne se gêne pas pour le dire: mettre le fond avant la forme, c'est donner la palme à un bègue ânonnant, écrit-il, car cette vénération du fond dont l'immense majorité des Canayens souffre est un reliquat de formation clérico-ecclésiastique. Et il termine: je comprends en ce sens SANS L'ADMETTRE! la primauté du fond sur la forme; je me ferais brûler vif avant de concéder (17 juin 1933).

Connaissant ce désaccord fondamental, on peut prévoir que Pelletier et DesRochers n'arriveront pas non plus à se mettre d'accord sur une définition de la poésie et de la critique. À l'utilité immédiate de la poésie selon Pelletier, DesRochers oppose son origine mystique, comme le poète d'ailleurs qui est essentiellement mystique, explique$\mathrm{t}$-il en se référant aux théories de l'abbé Bremond ${ }^{6}$ : un poète qui ne croit pas en Dieu ou qui n'est pas au moins déiste, ça n'existe pas (27 avril 1930). S'appuyant sur des critères impressionnistes et humanistes - qui doivent plus à sa formation académique qu'il ne veut bien l'admettre -, Pèlletier continue à parler de vers et de poèmes qui doivent avoir une âme, et n'être pas simplement un procédé d'écriture (7 septembre 1932), alors que DesRochers insiste sur la poésie comme travail de la forme, comme moyen d'exprimer le plus de sensation, de suggestion, de pensée même avec un minimum de mots (15 septembre 1932). L'opposition persiste tout au long de la correspondance, mais l'accrochage le plus sérieux se produit en 1935, lorsque le poète soumet pour publication au directeur des Idées sa conférence portant sur "L'avenir de la poésie en Canada français". Dans une lettre où il se révèle farouchement et étroitement nationaliste, Pelletier prend à parti ce qu'il appelle le pan-américanisme de langue anglaise de DesRochers, et il lui demande d'apporter des modifications majeures à son texte avant même de le faire lire par le comité de rédaction de la revue (23 février 1935). Un froid s'installe sûrement entre les deux hommes à la suite de cette lettre (nous n'avons pas la copie de la réponse de DesRochers), car la correspondance s'interrompt pendant un an avant de reprendre le 14 février

6 Voir en particulier la Poésie pure (1926) et Prière et poésie (1926) de l'abbé Henri Bremond. 
1936. L'article paraîtra finalement dans deux numéros successifs des Idées, en juillet et en août 1936.

Sur le sujet de la critique littéraire, le désaccord n'est pas aussi marqué au début de la correspondance, mais il ne tarde pas à apparaître, tant les tempéraments des deux écrivains sont aux antipodes. Après avoir publié deux recueils de poèmes, DesRochers considère qu'il ferait un bien meilleur critique que poète, mais il explique que ce sont les circonstances et des défis personnels qui l'ont amené à écrire de la poésie (27 avril 1930). Pelletier est aussi d'avis que la critique du poète de l'Orford est originale et pertinente, et cela surtout après la parution de Paragraphes (1931), mais il differe d'opinion sur le genre de critique que pratique DesRochers. Celui-ci, à l'exemple de Louis Dantin, met l'accent sur l'empathie et la sympathie plutôt que sur la distance critique et l'éreintement; en un mot, il préferre une critique positive et constructive à une appréciation négative, à l'instar de la critique d'identification que privilégie Dantin (2 mars 1931). Il parle lui-même d'une critique impressionniste dans son avant-propos de Paragraphes ${ }^{7}$. Mais Pelletier ne le voit pas du même œil. Aussitôt la lecture du recueil d'«interviews littéraires" terminée, il démontre dans une longue lettre à DesRochers (11 juillet 1931), preuves à l'appui, que sa critique n'est pas impressionniste, mais essentiellement didactique et dogmatique. Toutefois, s'empresse-t-il d'ajouter, c'est la diversité de toutes ces sentences dogmatiques - et il $y$ en a plus dans ce livre que je n'en ai vu encore dans aucun autre lịvre de critique - qui fait l'intérêt de Paragraphes (11 juillet 1931), et cela vaudra plus tard à DesRochers le titre de critique le plus compétent en poésie (14 février 1936).

Les exemples cités jusqu'ici montrent que le ton est tout de même resté modéré dans les échanges sur la critique, malgré les vues divergentes des deux correspondants. Mais la coupe déborde lorsque Pelletier fait une évaluation sévère de l'Almanach littéraire de l'Est, le supplément publié par DesRochers dans la Tribune d'août 1932 et adressé à son ami montréalais. Par retour du courrier, Pelletier qualifie les textes des Écrivains de l'Est d'exercices littéraires, sauf les vers de DesRochers bien sûr (31 août 1932). Celui-ci, qui a mis beaucoup de temps et d'énergie à préparer ce troisième supplément annuel du quotidien de Sherbrooke, encaisse d'abord le coup, puis réplique dans une lettre où il juge la critique de Pelletier d'une sévérité outrancière (5 septembre 1932). En fait, conclut DesRochers, du critique le mieux outillé de ma génération que tu étais [...] tu es en train de te casser le cou en surdéveloppant ton sens critique. Pelletier répond aussitôt qu'il n'accepte pas ce jugement, car

7 Alfred DesRochers, Paragraphes. (Interviews littéraires), Montréal, Librairie d'action canadienne-française, 1931, p. 7-11. 
nous manquons tous désespérément, nous les Canayens, de sens critique, et il cite à titre d'exemples la pauvreté apocalyptique des écritś critiques de Louis Dantin, Mgr Camille Roy, Maurice Hébert, Séraphin Marion, et il inclut même dans cette liste son propre recueil critique et celui de DesRochers ( 7 septembre 1932) ${ }^{8}$. Pour la première fois, le poète de Sherbrooke attaquait de front la sévérité critique de l'auteur de Carquois, mais ce ne sera pas la dernière, car cette sévérité créera au chroniqueur du Canada de nombreux ennemis dans le milieu littéraire. Les lettres où Pelletier prend à partie Clément Marchand (10 octobre 1932), Berthelot Brunet (12 décembre 1933) et Gonzalve Désaulniers (19 février 1934) en sont de bons exemples, sans parler : de la lettre-invective envoyée à Monsieur Bruchési... ou Mademoiselle qui constitue un sommet en matière d'attaque personnelle ${ }^{9}$.

Ce qui frappe le plus les lecteurs contemporains dans ces lettres portant sur la critique littéraire, c'est tout ce que les correspondants écrivent officieusement, mais qu'ils ne publient presque jamais officiellement ou intégralement dans leurs articles de revues et de journaux ou dans leurs livres de critique. À la fin de la longue lettre (déjà citée) où il qualifie la critique de Paragrephes de didactique et dogmatique, Pelletier écrit: Au revoir. Je ne te dirai pas toutes ces bêtises en public. (11 juillet 1931) Et en effet, le ton s'est adouci, de même que les formules de sa critique du recueil, en passant de la lettre au compte rendu publié dans Egrappages ${ }^{10}$. Autre exemple: en 1931, dans une lettre évoquant une de ses chroniques du Canada, Pelletier révèle ses préférences en ce qui a trait aux recueils de poèmes de Jovette Bernier et d'Éva Senécal, mais il avoue du même souffle que son rédacteur en chef, Olivar Asselin nous donne beaucoup de liberté, mais [...] ne veut pas qu'on s'aplatisse, car il vise à la distinction de son journal (5 octobre 1931).

On voit ainsi qu'entre ce que les écrivains et les critiques livraient dans leur correspondance et ce qui paraissait dans les revues, il y avait souvent une nette différence. Un cas extrême d'autocensure - mais non le seul - est cette lettre (3 mars 1931) dans laquelle DesRochers soumet à Pelletier son compte rendu de Carquois et lui demande en quelque sorte son approbation avant de le publier dans la Tribune. Il

8 Cependant, il se garde bien de nommer Valdombre, car il a un faible depuis le début pour ce type, parce que c'est un type, [...] très intelligent et parfois génial (voir sa lettre du 11 avril 1930). C'est d'ailleurs Pelletier qui publiera Un homme et son péché en 1933.

9 Pelletier envoie une copie de cette lettre de deux pages, datée du 5 novembre 1931, à DesRochers qui l'a conservée dans son fonds déposé aux Archives nationales du Québec à Sherbrooke.

10 "Paragraphes d'Alfred DesRochers", Albert Pelletier, Égrappages, Montréal Éditions Albert Lévesque, 1933, p. 114-122. 
y a d'autres lettres tout aussi intéressantes où les deux correspondants discutent des mérites respectifs des deux recueils de DesRochers pour savoir lequel est supérieur ( 7 mars et 25 juin 1930), ou de la nature du talent poétique de DesRochers comparé à celui de Robert Choquette (23 avril 1930), ou encore des raisons de publier ou de ne pas publier les quarante premières strophes de ce qui paraîtra trente ans plus tard sous le titre le Retour de Titus ( 9 avril et 10 juin 1933).

\section{La Crise et ses répercussions}

Dès les années 1931-1932, et surtout en 1933, la correspondance met l'accent sur les sujets socio-politiques et économiques, conséquence inévitable de la Crise qui se fait sentir en Amérique du Nord après le krach financier de 1929. La critique du système d'enseignement collégial - véritable bête noire de Pelletier - est un des premiers thèmes abordés, mais d'autres sujets mettront aux prises un Pelletier de plus en plus polémiste et un DesRochers souvent sur la défensive face aux prises de position catégoriques de l'auteur de Carquois. Le 27 février 1931, Pelletier écrit une longue lettre entièrement consacrée aux problèmes de l'enseignement, du cours classique dépassé et à réformer et de la formation des prêtres enseignants qui est à revoir de fond en comble. DesRochers est compté parmi les chanceux qui ont pu échapper au système, selon Pelletier, car lui, Asselin et Parizeau sont des autodidactes, alors que l'abbé Groulx et le père Lamarche sont des insubordonnés, ce qui les a sauvés. Tout au long de l'hiver 1931, les lettres des deux correspondants reviennent sur le sujet jusqu'à ce qu'ils aboutissent aux questions de la langue et de la culture nationales.

Il fallait s'y attendre, car Pelletier a publié Carquois en janvier 1931, son premier recueil d'articles (parus pour la plupart dans la Revue moderne), dont l'introduction est un essai intitulé "Littérature nationale et nationalisme littéraire" 11 . Or cet essai, dans lequel Pelletier prend position en faveur d'une littérature et d'une langue authentiquement "canadiennes", met le feu aux poudres. Il fait aussitôt l'objet de commentaires passionnés et suscite même quelques querelles, dont la plus célèbre est celle qui mit aux prises les deux critiques les plus influents de l'époque: l'aîné Louis Dantin, dont la réputation n'était plus à faire, et le même Pelletier, qui est en train de s'en tailler une. La position de DesRochers est délicate, car il entretient une correspondance suivie avec les deux critiques et on lui demande en quelque sorte de jouer le rôle de médiateur. Les deux adversaires ayant présenté et défendu leur position respective au cours de l'hiver et du printemps 1931, Pelletier écrit à DesRochers en juin:

11 Albert Pelletier, Carquois, Montréal, Librairie d'action canadienne-française, 1931, p. 7-33. 
Dantin s'est toqué sur cette idée ridicule et stupide: être canadien, ça veut dire ignorer absolument tout ce qui se passe, se fait, s'écrit, se pense et se sent à l'étranger. Comme il s'est toqué sur cette autre idée ridicule et stupide: le vocabulaire canadien se compose presque exclusivement d'anglicismes et de canadianismes de filles de facteries. [...] Je lui ai écrit dans ce sens tout l'hiver dernier, mon cher DesRochers. Et tu vois que cette idée ne s'est pas modifiée d'un iota. [...] après ce que je lui ai écrit, sa petite note de l'Avenir du Nord [sur l'essai Légionnaire !... d'Henri Pouliot, 5 juin 1931] est, je le répète, une saloperie. Tu auras sans doute plus de chances que moi de faire accepter tes idées qui sont le bon sens même, car il n'a pas de raison de t'en vouloir autant qu'à moi. (30 juin 1931)

Que DesRochers réussisse ou non à convaincre Dantin, peu importe à Pelletier finalement, car il n'en démord pas: il nous est impossible, à cause de notre histoire, de notre milieu, de nos aspirations naturelles, de notre climat, de nos conditions de vie, etc., de nous assimiler le génie de la race française. C'est même, selon lui, un illogisme historique, une contradiction fondamentale, une utopie! Mieux vaut d'abord s'enraciner dans le génie de la nation canadienne et, après, mais après seulement, nous poürons nous assimiler avec profit la culture française et toutes les autres cultures $d u$ monde (24 juillet 1931). La position de médiateur de DesRochers est d'autant plus délicate que l'option franchement "canadienne" de Pelletier est largement partagée par le poète d'À l'ombre de l'Orford.

Mais Pelletier est un homme aux idées plus arrêtées et, on a vite fait de le constater en lisant la correspondance, au caractère beaucoup plus conservateur que DesRochers. La quarantaine de lettres échangées au cours de l'année 1933 nous en fournit une preuve incontestable ${ }^{12}$. C'est la Crise, bien entendu, qui amène les correspondants à parler de philosophie politique et de système économique. C'est à un DesRochers fondamentalement libéral et démocrate que Pelletier, homme de droite, expose ses idées. Celui-ci croit, comme Flaubert au XIX ${ }^{\mathrm{e}}$ siècle et comme beaucoup d'intellectuels canadiensfrançais des années trente, à un mandarinat intellectuel et politique comme meilleure forme de gouvernement, un mandarinat qui pourrait aller jusqu'au despotisme éclairé ou à la dictature héréditaire:

J'en suis rendu à penser que [...] dans le gouvernement individuel, familial, économique ou national, les pieds ne prennent jamais avantageusement la place du cerveau. C'est

12 Les lettres se suivent à un rythme de trois ou quatre par mois et il s'agit souvent de longues lettres qui devaient demander plusieurs heures de réflexion et de dactylographie. Le fonds Alfred DesRochers contient 21 lettres de Pelletier et seulement trois, mais trois importantes lettres de DesRochers, pour l'année 1933. 
pour cela que j'ai plus confiance en n'importe quelle dictature, même mauvaise, plus confiance surtout en une dictature héréditaire, qu'en toute forme de gouvernement dirigée par la masse. Il y aura des abus et du favoritisme, j'en conviens, mais seulement pour quelques-uns qu'il est d'autant plus facile de tenir dans des limites convenables. [...] Dans un régime démocratique, au contraire, tous les ministres et députés sont invités à abuser, et tous les amis de ministres et de députés. [...] En fait, sinon en théorie, le peuple n'a rien de plus à voir dans ce gouvernement que sous un régime de monarchie absolue. (21 juin 1933)

Étant anti-démocrate, comme le note DesRochers lui-même, Pelletier est contre le suffrage universel et pour le régime du propriétisme, car il tend à accroître la production et assure à l'individu la plus grande somme de liberté (5 juillet 1933). En fait, dans le contexte des années trente, le jeune Pelletier se révèle non seulement antidémocrate, mais anti-socialiste et anti-communiste, et certaines de ses positions en faveur d'une société stricte de law and order le rapprochent plus du fascisme que de tout autre régime politique:

[...] au lieu de croire que les dictatures de Mussolini, Hitler, Roosevelt, Dolfuss sont un grand mal et un acheminement vers le communisme, je crois qu'ils [sic] sont un premier pas vers une notion plus intelligente du gouvernement, et qu'il faudrait bénir même l'ignorance fanatique d'Hitler s'il donne un croc en jambe au suffrage universel ou à 60 millions d'ignorances fanatiques. (21 juin 1933)

DesRochers répond à chacune des prises de position de Pelletier par ses propres prises de position, en personne ou par écrit. Il se rend par exemple chez les Pelletier le 8 juillet 1933 et discute monnaie, propriétisme, démocratie avec lui et Lucien Parizeau toute la soirée. De retour à Sherbrooke, quelques jours plus tard, il écrit: Vous aviez d'excellents arguments - qui ne m'ont pas convaincu. Mon inaptitude à m'exprimer oralement m'a empêché de faire valoir certaines de mes vues. Ma littérature sera-t-elle plus claire? - Essayons. (14 juillet 1933) Pendant le mois de juillet de cette même année, les deux hommes se plongent dans différents livres sur la théorie monétaire ét échangent six longues lettres sur le sujet, en plus de leur rencontre du début du mois. En 1934 et 1935, Pelletier discute encore, bien que moins souvent, des causes du désordre économique (nous récoltons les fruits poussés sur notre caractère de nomades et de coureurs d'aventures providentielles à l'instar des sauvages) et des moyens d'y remédier en rétablissant une échelle des valeurs humaines basée sur l'agriculture, l'épaulement de toute la structure sociale (25 octobre 1934).

À l'approche des élections provinciales, Pelletier prévient DesRochers de la défaite prochaine du premier ministre Alexandre 
Taschereau, car il n'est pas un dictateur alors qu'il le peut (20 février 1935). Il n'est donc pas surpris, en août 1936, de la perte du pouvoir du Parti libéral, au profit de l'Union nationale de Maurice Duplessis; mais ce qui nous frappe aujourd'hui dans la lettre qu'il fait parvenir à DesRochers à cette occasion, ce sont les dernières lignes qui marquent un revirement d'attitude:

J'ai eu beau pistonner Asselin et Parizeau qui me semblaient les plus aptes à comprendre, ils ont marché aussi à l'aveugle que Paul Gouin, que les Jeune-Canada, que les jeunesses patriotes, que toutes les marionnettes que des gens habiles agitaient. Car tous ces groupements et bien d'autres, ainsi que le dada corporatiste, sont sortis de la même usine et toujours pour détourner l'attention. Il me faut conclure que c'est encore le clergé qui reste notre classe la plus intelligente, et parmi le clergé, la Société des Jésuites. Cela règle toutes mes hésitations: j’envoie Gilles au collège Sainte-Marie la semaine prochaine. (23 août 1936)

Et quel revirement! Après avoir pesté contre l'enseignement clérical des collèges classiques pendant des années, voilà que Pelletier décide finalement d'envoyer son fils chez les jésuites.

\section{L'édition littéraire: une question d'apostolat}

Ce n'est que vers 1933-1934 que Pelletier et DesRochers se mettent à discuter sérieusement des problèmes de l'édition littéraire au Québec. Deux raisons en particulier expliquent leur intérêt pour l'édition. Au début des années trente, les deux auteurs publient chez le même éditeur, la Librairie d'action canadienne-française (plus tard les Éditions Albert Lévesque), qui a le vent dans les voiles et qui détient le quasi-monopole de l'édition littéraire québécoise; vers 1933-1934, alors que la maison Lévesque connaît des ennuis et commence à péricliter, Pelletier et DesRochers sont amenés à se demander comment un éditeur littéraire peut survivre dans le contexte d'un marché restreint et déprimé comme celui des années trente au Québec. Puis les deux jeunes écrivains, une fois leurs premiers livres publiés, sont de moins en moins satisfaits de leur contrat, de la promotion et de la diffusion de leurs titres parus chez Lévesque, à telle enseigne que finalement ils tenteront de mettre sur pied leur propre maison d'édition.

En fait, DesRochers a déjà fait paraître deux recueils à compte d'auteur à la fin des années vingt, l'Offrande aux vierges folles (1929), imprimé à Coaticook, et À l'ombre de l'Orford (1929), imprimé à Sherbrooke, avant de lancer commercialement un premier livre à Montréal en $1930^{13}$. Contrairement à Pelletier, il a donc, au début

13 Il s'agit d'une réédition d'À l'ombre de l'Orford, augmentée d'un choix de treize poèmes tirés de l'Offrande aux vierges folles. Le recueil est préfacé par Alphonse 
des années trente, une expérience concrète des différentes étapes de l'édition, de la diffusion et de la vente d'un recueil. C'est ce qui explique que dans deux lettres du mois de février 1931, Pelletier le félicite d'avoir réussi à placer une soixantaine d'exemplaires de l'Orford auprès du gouvernement du Québec ${ }^{14}$ alors que lui, pauvre comme Job, n'a pas vendu un seul exemplaire de Carquois après s'être fait seriner depuis près d'un an que [son] livre paraîtra demain (14 et 27 février 1931). Les deux correspondants reviennent sur le sujet à quelques reprises par la suite puis, de retour d'une visite chez les DesRochers au début de juin 1933, Pelletier décide de présenter son projet à son ami:
Je sollicite pour les Elzévirs, dont tu peux être si tu veux sans débourser un sou. Cette idée m'est venue à la suite d'une con- versation à Sherbrooke, où tu émettais l'avis que 5 ou 6 au- teurs devraient s'engager jusqu'à concurrence de $100 \$$ chacun à supporter les frais de publication des livres qui en valent la peine. (10 juin 1933)

Comme il n'est pas facile de trouver des jeunes qui ont les moyens financiers de prendre cet engagement et, comme de surcroît, DesRochers se trouve à cent soixante kilomètres de Montréal, Pelletier fait appel à Lucien Parizeau, un jeune journaliste du Canada, pour mettre sur pied les Elzévirs (devenus plus tard les Éditions du Totem ${ }^{15}$ ). Il s'agit essentiellement d'une entreprise coopérative où les deux

Désilets et publié par la Librairie d'action canadienne-française (Montréal, 1930, 157 p.).

14 Les lettres des 14 et 27 février 1931 de Pelletier à DesRochers confirment ce que l'éditeur Clément Marchand nous a déjà dit en interview: un des débouchés pour la vente d'un livre "littéraire" et une façon de se constituer un petit capital pour en publier d'autres, c'etait d'en placer un certains nombre d'exemplaires auprès de différents ministères du gouvernement du Québec et surtout auprès du secrétaire de la province, Louis-Athanase David. DesRochers a décidé de faire une réimpression de l'édition originale d'À l'ombre de l'Orford, dès novembre 1929, pour répondre à la commande du Secrétariat de la Province (commande qu'il avait lui-même sollicitée). Au sujet de ces subventions de l'État aux auteurs, et non aux éditeurs, voir notre article portant sur «L'éditeur et l'édition de poésie, 19401960 ", dans l'Institution littéraire, sous la direction de Maurice Lemire, Québec, Institut québécois de recherche sur la culture et Centre de recherche en littérature québécoise, 1986, p. 181-189.

15 Elzévir est le nom d'une famille de libraires et d'imprimeurs hollandais installés à Leyde, puis a La Haye, Copenhague, Utrecht, Amsterdam, aux XVI et XVII siècles. Le nom est employé pour parler d'un livre [un elzevir] imprimé en Hollande par les Elzevir [...], ou par leurs imitateurs et, par extension, pour désigner un caractère à empattements triangulaires, proche du type employé par les Elzévir ou leur petit format (in-12) qui les a rendus célèbres (le Petit Robert). Sans doute Pelletier considérait-il le nom de sa maison, les Elzévirs, trop érudit, difficile à prononcer et faisant peut-être trop européen, après la parution de Walt Whitman de Rosaire Dion-Lévesque. Toujours est-il qu'en janvier 1934, il utilise un nouveau papier à lettres, avec comme en-tête les Éditions du Totem, sans 
fondateurs feraient le plus gros du travail et se partageraient $75 \%$ des bénéfices, alors que des associés à Québec, à Ottawa et à Sherbrooke, chargés de trouver des manuscrits et d'aider à la promotion et à la diffusion des livres, récolteraient le reste. Pelletier a même demandé à l'imprimeur Rioux, de la Parole de Drummondville, de se procurer un caractère exclusif (le caractère Estienne) pour la maison d'édition et pour une revue que nous avons l'intention de publier au bout d'un an (10 juin 1933). Dans une lettre écrite trois jours plus tard, Pelletier expose en détail le fonctionnement de la maison et la répartition des risques, des bénéfices et des tâches d'une édition coopérative comparée à un éditeur comme Albert Lévesque par exemple, et il demande à DesRochers de collaborer comme membre associé.

DesRochers s'empresse de répondre à l'offre de son ami et évalue le projet de façon réaliste et pratique. En estimant que chaque volume coûterait en moyenne $40 \varnothing$ à produire et en établissant le prix de vente au détail à 1 \$ le volume, il resterait $1 \%$ à distribuer aux lanceurs, calcule DesRochers soit : production, $40 \phi$; frais généraux, $16 \phi$; remise aux libraires, $40 \phi ; 75 \%$ des profits nets, $3 \phi$, et dividendes, 14 . À ce compte et étant donné qu'il faudrait immobiliser un capital de quelque $500 \$$ au moins par année, DesRochers conclut sa lettre par le commentaire suivant: L'édition au Canada, après l'avoir étudié passablement, me semble une question d'apostolat, non d'affaires, et seules les communautés rendent l'apostolat possible... (17 juin 1933) ${ }^{16}$ Pelletier réplique aussitôt qu'il est d'accord avec les calculs de DesRochers, mais qu'il lance quand même les Elzévirs, à la fin du mois, avec le recueil de Rosaire Dion (des traductions de Walt Whitman), qu'il entend publier six livres par année (DesRochers, Medjé Vézina, Harvey, Léo Parizeau, Asselin, etc.), plus une revue libre, et qu'il compte enfin sur 100 abonnés pour le premier livre, au moins 300 pour les autres, et plus de 700 comme objectif à long terme pour dépasser le nombre atteint par Louis Carrier des Éditions du Mercure (lettre non datée, vers le 19 juin 1933).

expliquer dans sa correspondance avec DesRochers pourquoi il a choisi ce nom à consonnance amérindienne. Mentionnons qu'à partir de cette même année 1934, Pelletier est seul à diriger la maison d'édition et ne mentionne plus son collaborateur Lucien Parizeau. C'est sous ce nom des Éditions du Totem qu'il fit paraître les romans de Grignon et Harvey, en 1933 et 1934.

16 Cette lettre (17 juin 1933) et quelques autres de la même époque nous aident à comprendre pourquoi, à cause de son travail de jour et de ses ressources financières limitées, DesRochers n'a pas fondé une véritable maison d'édition à Sherbrooke. Il a créé une association d'auteurs des cantons de l'Est, «les Écrivains de l'Est », et il a fait un peu d'édition en se servant des presses de la Tribune (son deuxième recueil et ceux de son ami Dantin, entre autres), il a dirigé pendant un an l'hebdomadaire l'Étoile de l'Est de Coaticook et il a mis sur pied un nouvel hebdomadaire à Sherbrooke, le Progrès de l'Est, qui a paru deux ou trois fois au début de 1935. Mais dans le cas d'un hebdo, il y a possibilité que l'affaire soit rentable, ce qui lui a toujours semblé impossible avec une maison d'édition. 
Il est à noter què c'est en juin 1933, deux mois après la parution chez Albert Lévesque de son recueil Égrappages, que Pelletier fonde sa maison d'édition. Il n'est pas sans se rendre compte que les Elzévirs semblent faire concurrence à Lévesque, d'autant que dans le monde de l'édition québécoise de cette époque, la fondation d'une nouvelle maison met aussitôt en danger les quelques éditeurs littéraires existants (Lévesque, Carrier, Garand). Pelletier a beau assurer à DesRochers qu'il ne publie pas les mêmes livres que Lévesque, qu'il ne vise pas la même clientèle, et surtout que les écrivains doivent prendre les devants et se prémunir contre la fermeture éventuelle de cet éditeur, il reste qu'il se sent mal à l'aise, d'autant plus que ses relations avec l'autre Albert sont mauvaises depuis au moins la publication de Carquois en $1931^{17}$. Quoi qu'il en soit, la maison de Pelletier semble répondre à un besoin puisqu'il rapporte au début de 1934 que les Éditions du Totem comptent déjà 284 abonnés, après la publication de seulement deux livres: Walt Whitman de Rosaire Dion, tiré à 3000 exemplaires (qui s'écoulent lentement), et Un homme et son péché de Claude-Henri Grignon (dont le premier tirage se vend très bien). Et cela malgré le fait, écrit-il à DesRochers, que j'ai envoyé 664 lettres avec formules d'abonnement à autant de maisons d'éducation et que pas une seule n'a répondu (11 janvier 1934). La revanche, pourrait-on dire ironiquement, ou en tout cas le véritable succès des Éditions du Totem viendra en avril 1934 avec la publication des Demi-civilisés de Jean-Charles Harvey (tirage de 3000 exemplaires), suivie aussitôt du décret du cardinal Villeneuve interdisant la vente et la lecture du roman dans le diocèse de Québec. Pelletier ne peut contenir sa joie dans une lettre adressée à DesRochers à la fin du mois d'avril:

Depuis, j'ai vendu 430 exemplaires à Montréal: 147 hier, et le reste cet avant-midi. Il ne m'en reste plus un seul exemplaire ici. Un boom! Une surprise! Une réclame comme il ne s'en est . jamais vu! Je me demande si, en conscience, je ne dois pas payer une commission au Cardinal comme agent de publicité.

17 D'abord Pelletier a reproché à Lévesque d'avoir reporté la publication de Carquois d'au moins un an, une fois le recueil paru, de ne pas faire assez de publicité et de ne pas pousser la vente du livre auprès du Secrétaire de la Province. Le froid entre les deux hommes est tel qu'en juin 1933, deux mois après le lancement d'́́grappages, le critique ne sait pas que son éditeur prépare, en plus du tirage régulier, un tirage de luxe de cent exemplaires de son deuxième livre. Pelletier n'est donc pas mécontent d'apprendre à DesRochers que Lévesque est indécis et [...] attend notre première édition pour décider ce qu'il pourra faire comme agent, partenaire ou collaborateur (lettre du 13 juin 1933). Par contre, il est' important de le souligner, c'est ce même Pelletier qui rejette en 1934 une proposition d'article de DesRochers dans lequel celui-ci veut s'en prendre publiquement à Lévesque dans les pages du premier numéro de la revue les Idées: $I l$ ne conviendrait pas, écrit-il au poète de Sherbrooke, d'attaquer dans un grand article Lévesque qui n'a pas d'organe pour se défendre. (22 décembre 1934) 
Québec en a acheté 900. [...] Mon cher DesRochers, écris donc des livres qui se fassent panacher d'un décret cardinalice! [...] Mais j'ai soumis les épreuves à trois prêtres montréalais qui en ont permis la publication. [...] Le livre d'Harvey, de l'avis de mes trois théologiens, et du mien, est essentiellement moralisateur. (28 avril 1934)

Ce roman, un des premiers best-sellers de l'édition littéraire moderne au Québec ${ }^{18}$, ajouté au succès d'Un homme et son péché de Grignon ${ }^{19}$, permet à Pelletier de voir plus grand et de consacrer plus de temps à la revue qu'il projette de créer depuis 1933. Faisons la part des choses toutefois: le jeune éditeur voit grand, mais pas assez pour pouvoir se permettre les coûts d'une publicité décente de ses livres dans les journaux et les revues à grand tirage. Son rôle, sa part de risque comme petit éditeur, écrit-il à DesRochers, consiste à dénicher des manuscrits et à publier des livres de bonne valeur, et il revient aux libraires de faire leur part dans la promotion et la publicité des auteurs et de leurs livres. Mais la grande majorité n'en fait rien, ajoute-t-il, car en ce pays, seuls les libraires qui vendent pour $80000 \$$ par année au gouvernement peuvent se permettre des frais de publicité (15 mars 1935). Pendant les premiers mois de l'année 1935, alors qu'il traite avec DesRochers de quelques lignes de publicité à insérer dans l'hebdomadaire le Progrès de l'Est ${ }^{20}$ et des remises qu'il est prêt à

18 Un autre cas est celui du roman l'Appel de la race d'Alonié de Lestres, pseudonyme de l'abbé Lionel Groulx. Le livre, publié en septembre 1922 par la Librairie d'action canadienne-française, est en réimpression dès cette année-là et encore en 1923. Lorsque le titre entre dans la collection du Nénuphar chez Fides en 1956, il en est à sa cinquième édition et plus de 15000 exemplaires ont été vendus. Il est à noter que dans le cas des Demi-civilisés, comme dans celui de l'Appel de la' race, il s'agit de romans à thèse publiés par des petites maisons qui éditent aussi toutes deux une revue de combat.

19 Nous savons par une lettre de Pelletier qu'en décembre 1934, lorsque Grignon rachète les droits de publication et le stock des invendus d'Un homme et son péché, 1800 exemplaires du roman, sur un tirage initial de 3000 , ont été écoulés en un an seulement (lettre du 26 février 1935).

20 À DesRochers qui accepte d'insérer dans le premier numéro de son hebdo de la publicité pour les livres des Éditions du Totem sauf le roman de Jean-Charles Harvey, Pelletier écrit en mars 1935: Tu as tort de ne pas annoncer des le premier numéro les Demi-civilisés. Nous sommes dans un pays où la moindre concession donne aux gens un "pied" extraordinaire, et ou le moindre acte de liberte, fait sans forfaiture, fait reculer pour un an tous les troupeaux de peureux. Pas un seul, curé ou non, ne meireproche d'annoncer le roman d'Harvey; et si je ne l'annonçais pas, tout le monde guetterait le moindre petit mot de travers pour me tomber dessus. On sait tout de suite de quel bois je me chauffe et on me fiche la paix. Pelletier et DesRochers confirment par ces lettres qu'il y a des pressions extérieures certaines qui s'exercent au Québec contre la liberté de parole des journalistes, des éditeurs et des écrivains de cette époque. Mais là ou le directeur des Éditions du Totem refuse de céder un pouce de terrain, DesRochers se met lui-même des fils aux pattes et aux mains en refusant d'annoncer les Demi-civilisés dès le premier numéro du Progrès de l'Est, sous prétexte de ménager les susceptibilités car, 
lui consentir pour la vente' de chaque volume, Pelletier parle aussi de la revue qu'il est en train de lancer. Il a trouvé un nom, le Réveil, une fréquence de parution (12 numéros par année) et un prix pour l'abonnement $(5 \$)$. Il veut des articles rigoureux, originaux, bien écrits, et il a même délimité le public lecteur qu'il vise: notre classe de lecteurs [...] ne sera pas celle du journal à faits divers et n'est donc pas intéressée aux stériles disputes et commentaires insignifiants [...] des 999 journaux et revues de la province (10 et 23 janvier 1935).

Si Pelletier est à ce point décidé, c'est qu'il connaît les possibilités de ses collaborateurs et les objectifs de sa revue:

Nous sommes un petit groupe à vouloir une vraie revue d'idées, non pas un vain dandinement autour de la pensée d'outremer. Il faut découvrir, circonscrire et percer les divers abcès qui nous réduisent à l'impotence, et suggérer les remèdes appropriés. Peux-tu dans quelque domaine (éducation, littérature, arts libéraux et arts manuels, politique, économie, finance, industrie, commerce, agriculture, arts ménagers, et le reste) trouver certaines causes précises de notre stagnation ou de notre recul, et suggérer des initiatives susceptibles de nous réveiller et de nous remettre en marche? (22 décembre 1934)

Une revue de combat et une revue d'idées donc, comme Pelletier l'appellera finalement ${ }^{21}$. Mais pas un journal de parti, car elle tiendra un discours en dehors de la politicaillerie des "rouges " à Québec et des «bleus " à Ottawa. Le jeune directeur sait bien que 1933 et surtout 1934 ont vu naitre un bon nombre de revues: l'Action nationale, l'Action universitaire, l'Ordre, la Relève, Vivre, mais ces périodiques révèlent à son avis l'état d'impuissance où la vie intellectuelle en est arrivée chez nous (22 décembre 1934). Il est donc prêt à se lancer dans l'aventure, car il sent qu'il y a encore beaucoup de lecteurs insatisfaits: les Montréalais du moins veulent du nouveau, de l'inédit, et c'est pour cela que les revues les désapointent (ibid.). Si l'on se fie au nombre d'abonnés mentionné dans une lettre à DesRochers un an après la fondation des Idées, Pelletier avait vu juste: 3000 abonnés en février 1936 et un tirage de 4000 exemplaires en août de la même année. En fait, il y a tellement de nouveaux abonnés au début de 1936 que le directeur revient sur sa décision de mettre un terme à son périodique après douze numéros et il en poursuivra la publication jusqu’à la veille

conclut Pelletier, le roman sera aussi dangereux dans quelques mois qu'il l'est au début de ton journal (lettre du 15 mars 1935).

21 Pelletier revient sur le nom d'abord choisi pour la revue car, explique-t-il à DesRochers, le Réveil est un titre dejà retenu et enregistré par d'autres. Alors la revue sera intitulé les Idées - ce qui veut dire que tout ce qui est pensée et vie de l'esprit y sera bien accueilli (10 janvier 1935). Le Réveil est un périodique qui a existé à Montréal, de mai 1915 à mars 1917. 
de la guerre, en juin $1939^{22}$. La dernière lettre de Pelletier à DesRochers est une lettre d'affaires qui négocie le coût d'une page d'annonce dans la revue pour un client de Sherbrooke (13 août 1936).

Avec le recul, on se rend compte que le cas du trop grand succès (pour un seul directeur qui assume à la fois les tâches de rédacteur en chef, d'administrateur, de publicitaire et de secrétaire de rédaction) de la revue les Idées (1935-1939) n'est pas surprenant ni unique. La revue Horizons de Clément Marchand, d'abord le Mauricien (TroisRivières, 1937-1939), a elle aussi connu un tel succès - plus de 6000 abonnés en 1940, selon une lettre de Marchand à DesRochers - que son jeune directeur a dû en cesser la publication, faute d'une infrastructure essentielle à son développement. Amérique française prendra la relève de ces deux revues et dotera la littérature québécoise de sa première revue de création littéraire de longue durée (1941-1955).

On le voit, il existe une importante demande d'information et de diffusion des idées au cours des années 30 et surtout à l'approche de la Seconde Guerre mondiale. Celle-ci marquera le dégel, le "réveil " du Québec en provoquant de nombreux bouleversements et en créant un nouveau climat de liberté d'expression dont les écrivains et les intellectuels seront les premiers à bénéficier. Malheureusement, la majorité des premiers combattants et des esprits contestataires qui furent à l'origine de ce renouveau, durement touchés par la Crise et à bout de souffle, ou bien cessent d'écrire ou bien ne publient plus de livres. Une nouvelle génération prend les commandes et les "individualistes" - selon le mot de DesRochers - qui ont animé l'entredeux-guerres sont balayés par le vent de changements qui souffle sur le Québec. C'est le cas de DesRochers, de Pelletier et de plusieurs autres: Harry Bernard, Jean-Charles Harvey, Jovette Bernier, Medjé Vézina, Éva Senécal, Claude-Henri Grignon. C'est en ce sens que DesRochers écrit, dans son sonnet à la mémoire du critique-animateuréditeur-directeur de revue Albert Pelletier, qu'il fit un sous-bois clair à la pensée auguste / Où les nouveaux s'avancent aujourd'hui. Le poète de l'Orford et l'auteur de Carquois comptent parmi les représentants de la race des précurseurs, des "éclaireurs" de la littérature québécoise, et leur importante correspondance nous rappelle la modernité des sujets de réflexion des écrivains des années 30.

22 Il peut sembler étrange qu'une revue continue de paraître, alors que son directeur avait décidé de cesser sa publication, parce qu'elle a trop de succès. C'est pourtant ce qui se produit pour les Idées en janvier 1936. Pelletier écrit à cette époque à DesRochers: Je suis plus fatigué que jamais, et mes nuits raccourcissent toujours, et je deviens fantome. Je devais finir les Idées en janvier, par un numéro spécial. Mais 714 abonnements nouveaux m'en ont empêché, et puis je voulais te donner l'occasion de parler aux cinq parties du monde: jai maintenant cinq abonnés au Tonkin, sept en Amérique du Sud, cinq en Afrique, un en Nouvelle-Zélande et une vingtaine en Europe. (14 février 1936) 